\title{
GAMMA-RAY CONSTRAINTS ON THE GALACTIC HALO
}

\author{
J. B. G. M. Bloemen
}

Leiden Observatory, P.O. Box 9513, 2300 RA Leiden, The Netherlands

\begin{abstract}
Some findings from recent COS-B analyses of the diffuse Galactic $\gamma$-ray emission are briefly summarized, with emphasis on their impact for our understanding of the Galactic cosmic-ray halo.

\section{INTRODUCTION}

It seems well established that the gaseous, magnetic-field, and cosmic-ray (CR) components of the interstellar medium (ISM) in our Galaxy, and several other galaxies, extend far beyond the disk into halo-type distributions. Little is known, however, about the actual size and structure of halos and also the physical relation between disk and halo is poorly understood. This disk-halo connection is of major importance for studies of interstellar processes and Galactic structure and evolution. Although the Galaxy at large may be in a hydrostatic equilibrium $/ 1,2 /$, it has been argued on both observational and theoretical grounds that a substantial mass and energy flow may exist between disk and halo and vice versa. The actual mode of mass and energy exchange will determine the characteristics of the ISM, including possibly those of CR propagation and confinement.
\end{abstract}

Quite opposite to the above scenario, direct CR observations near Earth have mostly been interpreted in terms of static, diffusion models. Although this approach turned out to be generally successful, it does not exclude dynamical models and there are in fact aspects that favor the latter (see e.g. Jones $/ 3 /$ ). Some new $\gamma$-ray results sketched below may give further insight.

\section{RADIAL COSMIC-RAY GRADIENT \& HALO DIMENSIONS}

Indirect size constraints on the CR halo in a standard diffusion model, as described by Ginzburg and Syrovatskii $/ 4 /$, can be obtained from $\gamma$-ray observations of the Galactic plane $/ 5 /$. This method was recently reconsidered by Bloemen and Dogiel /6/. They assumed a Galacto-centric distribution of CR sources that resembles the distribution of supernova remnants (SNR's) and investigated whether the radial CR density distribution that follows from the diffusion model, for different choices of the halo dimensions, is consistent with the CR distribution derived from the COS-B $\gamma$-ray data $/ 7 /$. The latter distribution holds for electrons with energies $\lesssim 1 \mathrm{GeV}$, which are responsible for the bremsstrahlung $\gamma$-ray emission, and for the $\mathrm{GeV}$ proton-nuclear component which is responsible for the $\pi^{\circ}$-decay $\gamma$-ray emission. Their findings are summarized in Fig. 1. It is clear that even a very large CR halo can barely explain the difference between the strong radial gradient of the source distribution and the observed weak CR density gradient.

Obviously, a SNR source distribution may not be the right choice. Furthermore, interpretation of the $\gamma$-ray data in terms of a ' $\mathrm{CR}$ gradient' may not be correct. The argument that can be made is simple. The observed $\gamma$-ray intensities trace only $\mathrm{CR}$ particles in those regions along the line-of-sight that contribute significantly to the total gas column density. Hence, the radial gradient of the volume-averaged CR density may be stronger, or weaker, than derived from $\gamma$-ray observations. It has been argued on theoretical grounds, for instance, that the CR density is correlated with matter density, although the scale on which this may occur is uncertain. Fichtel and collaborators (e.g. /8/ and references therein) considered a model with CR-matter coupling on the scale of spiral arms and found good overall agreement with $\gamma$-ray observations. Preliminary results from a study by Melisse and Bloemen /9/ show that CR-matter coupling on even smaller scales (typically $10-100 \mathrm{pc}$, so the scale of large clouds and cloud 'complexes) cannot be excluded by the COS-B observations. This is illustrated in Fig. 2. In this modelling no large-scale CR gradient is needed at all. Evidently, inferences regarding the Galactic halo from $\mathrm{CR}$ gradients cannot be very compelling at this stage. Higher resolution $\gamma$-ray data are needed. 


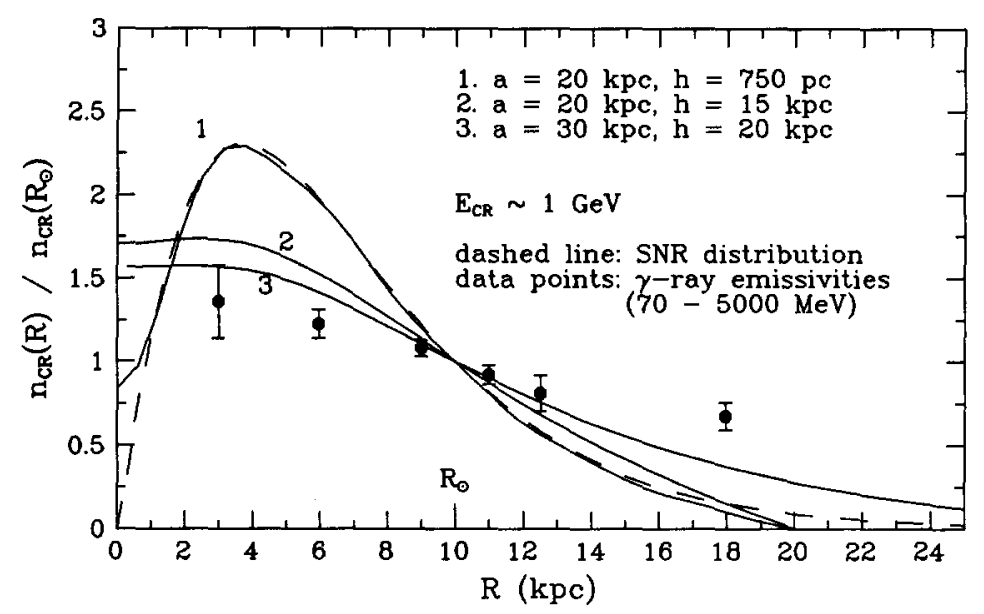

Fig. 1. Examples /6/ of the Galacto-centric CR density distribution in a diffusion model with a SNR-like CR source distribution and different halo dimensions ( $a$ : halo radius in the Galactic plane; $h$ : half thickness). The data points indicate the $\gamma$-ray emissivities ( $70 \mathrm{MeV}-5 \mathrm{GeV}$ ) derived by Strong et al./7/ from the COS-B data. All quantities are normalized at the radius of the solar circle.

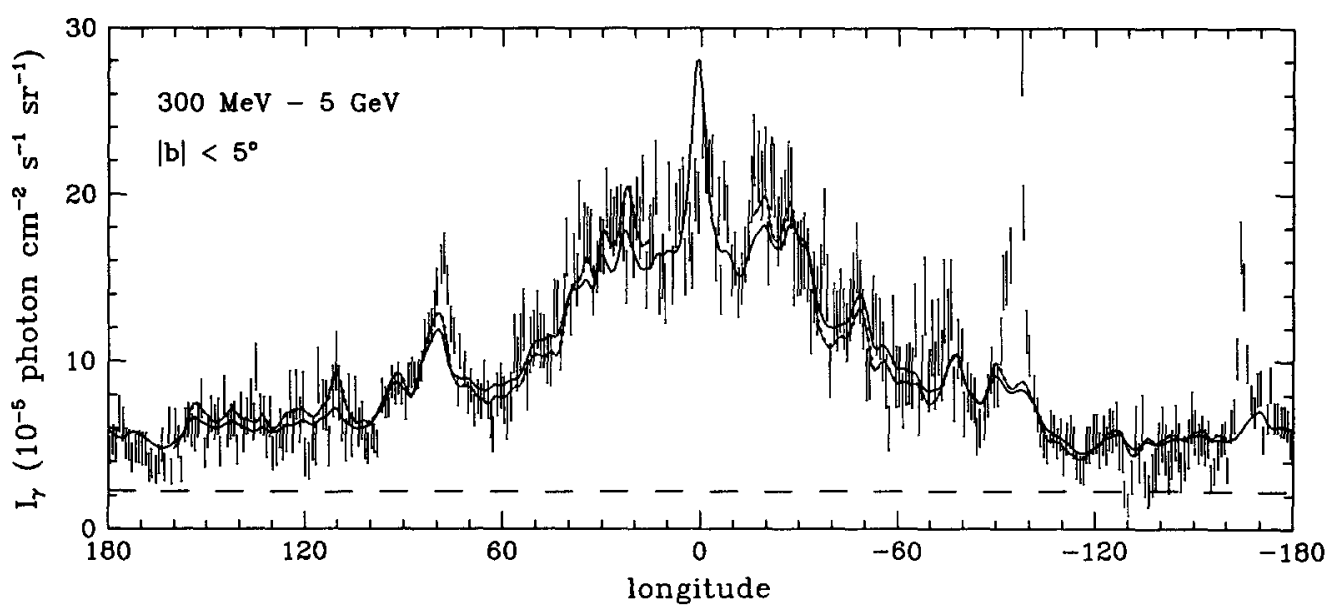

Fig. 2. Longitude distributions of the COS-B observations (error bars) and the predictions from the 'CR gradient model' of Strong et al./7/ (full line) and the 'CR-matter coupling model' of Melisse and Bloemen $/ 9 /$ (dotted line). The latter is based on the assumption that $\rho_{\mathrm{CR}} \propto \rho_{\mathrm{gas}}^{\alpha} ; \alpha=0.5$ was used for the specific distribution shown here.

\section{THE INVERSE-COMPTON GAMMA-RAY HALO}

Gamma radiation that originates at large distances from the Galactic plane can be expected to be produced predominantly by inverse-Compton (IC) scattering of relativistic electrons on low-energy photons (optical, infrared, and $2.7 \mathrm{~K} \mathrm{relic)}$. The gas densities here are too small to expect significant bremsstrahlung and $\pi^{\circ}$-decay contributions, which dominate the $\gamma$-ray emission from the Galactic disk. This IC $\gamma$-ray halo may already have been detected; there is increasing evidence that almost half of the observed $\gamma$-ray intensities at intermediate latitudes $\left(5^{\circ} \lesssim|b| \lesssim 20^{\circ}\right)$ in the inner-Galaxy direction cannot be explained by CR-matter interactions (see review by Bloemen /10/). This is shown in Fig. 3 for the energy range $70-150 \mathrm{MeV}$; the excess is most pronounced at these low energies, although it is clearly visible at higher energies as well.

The typical electron energy for IC emission at a $\gamma$-ray energy $E_{\gamma}$ is given by $E_{e} \simeq m_{e} c^{2}\left(E_{\gamma} / \varepsilon_{p h}\right)^{0.5}$, where $\varepsilon_{p h}$ is the average energy of the target photons. For the COS-B energy range $(\sim 50 \mathrm{MeV}-5 \mathrm{GeV})$ and $\varepsilon_{p h}(2.7 \mathrm{~K}) \simeq 6.3 \times 10^{-4} \mathrm{eV}$ (which dominates beyond a few kpc from the Galactic disk) this gives $E_{\mathrm{e}} \gtrsim 100$ $\mathrm{GeV}$. These energetic electrons suffer severe energy losses and fill therefore only a small part of the halo $(\sim 20-30 \%)$. Nevertheless, Bloemen and Dogiel /6/found that the scattering of these electrons on the $2.7 \mathrm{~K}$ background leads to excessive IC emission if the CR halo at $\mathrm{GeV}$ energies is as large as suggested by the observed weak CR gradient. Toward the inner Galaxy at medium latitudes, for instance, the predicted IC intensities are $2-4$ times higher than the average excess intensity visible in Fig. 3. This discrepancy is of course even larger if the optical and infrared photon fields would be taken into account. On the other hand, there is some flexibility in the model predictions because these are normalized to the electron spectrum near Earth, which is not accurately measured yet at these high energies. 


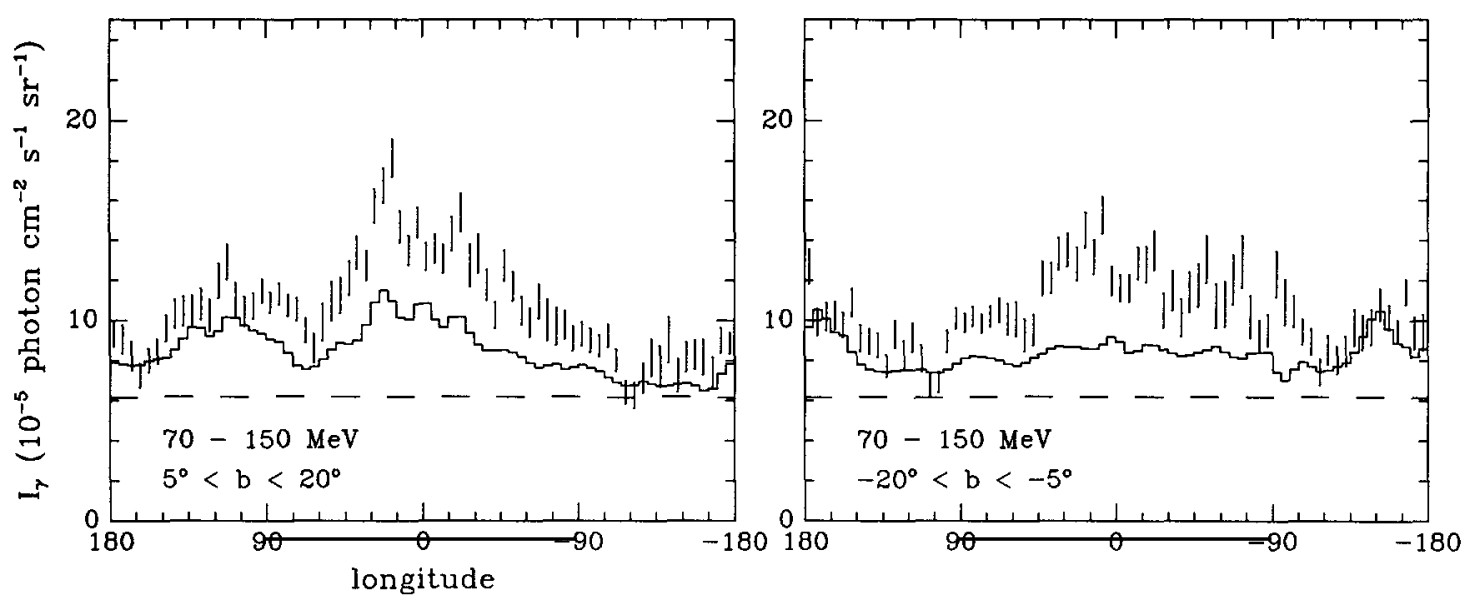

Fig. 3. Longitude distributions of the COS-B observations above and below the Galactic disk (error bars) and the model predictions from $C R$-matter interactions (an extension of the Galactic-disk model of Strong et al. $/ 7 /$ ). A broad intensity excess in the general direction of the inner Galaxy is evident.

\section{SPECTRAL BEHAVIOUR OF THE HALO}

Recent spectral studies of the diffuse $\gamma$-ray and radio-continuum emission of the Galaxy by Bloemen et al. /11/ and Reich and Reich /12/ show evidence for large-scale spectral variations which are hard to reconcile with CR diffusion models. These new results concern particularly the spectral-index distribution as a function of Galactic latitude. Reich and Reich /12/ determined a spectral-index map of radio-continuum surveys at 408 and $1420 \mathrm{MHz}$ and found a spectral flattening with increasing latitude, particularly in the general direction of the outer Galaxy. A spectral steepening due to electron energy losses is expected in a standard diffusion model. At lower frequencies a weak steepening has indeed been found (see e.g. review by Lawson et al./13/). The COS-B observations in the energy range $300 \mathrm{MeV}-6 \mathrm{GeV}$ show a similar effect: a spectral hardening with increasing latitude in the outer-Galaxy direction but no significant spectral changes in the inner-Galaxy direction /11/. Fig. 4 illustrates this finding in the form of latitude distributions of the ratio between the intensities in the $800 \mathrm{MeV}-6 \mathrm{GeV}$ and $300 \mathrm{MeV}-800 \mathrm{MeV}$ intervals.

The flattening of the $\gamma$-ray spectrum cannot be explained by CR diffusion models if the observed emission originates largely from CR-matter interactions. The IC contribution from a halo might however lead to such an effect. This follows from the fact that the $\pi^{\circ}$-decay $\gamma$-ray spectrum converges to a power-law spectrum at $E_{\gamma} \gtrsim 1 \mathrm{GeV}$ with a spectral index equal to that of the CR proton-nuclear spectrum above $\sim 10 \mathrm{GeV}$ ( 2.7 near Earth), whereas the IC spectrum is approximately given by $E_{\gamma}^{-[\Gamma+1] / 2}$ for an $E_{e}^{-\Gamma}$ electron spectrum (near Earth, $[\Gamma+1] / 2 \simeq 2$ for $E_{e} \gtrsim 10 \mathrm{GeV}$ ). Bloemen and Dogiel /6/ studied this possibility in the framework of the diffusion model with a large halo as suggested by the weak CR gradient (radius 30 $\mathrm{kpc}$ and half thickness $20 \mathrm{kpc}$ ). Their model predictions are included in Fig. 4. A weak spectral flattening with latitude can indeed be seen, but there is no significant difference between the inner- and outer-Galaxy directions and the observed effect (in the outer-Galaxy) is clearly much stronger. The predicted flattening with latitude is weaker than anticipated mainly because of the strong radiation losses for the high-energy electrons, which lead to a steepening of the IC spectrum with increasing distance to the Galactic plane (illustrated in Fig. 5).

A dynamical halo model $/ 3,14,15,16,17 /$ may provide a viable explanation. The competition of spatial diffusion, convection, adiabatic deceleration, and (electron) radiation losses in such a model may in principle lead to the observed effects, but the problem is very complex. Reich and Reich /12/ and Bloemen et al. /11/ noted that the findings are at least to a large extent in agreement with the asymptotic spectral predictions of the galactic-wind model given by Lerche and Schlickeiser $/ 14,15 /$. In this scenario, the $\gamma$-ray spectral variations are not related to IC emission, but are due to spectral-index changes of the CR proton-nuclear component at $\mathrm{GeV}$ energies (responsible for $\pi^{\circ}$-decay emission). This interpretation requires that a significant fraction of the $\gamma$-ray emission observed at medium latitudes originates from CR-matter interactions beyond several hundred parsecs from the plane, which may indeed be the case (see $/ 11 /)$.

In conclusion, a diffusion model with an extensive $\mathrm{CR}$ halo may explain reasonably well the observed weak $\mathrm{CR}$ gradient and the observed $\gamma$-ray excess at medium latitudes in the inner-Galaxy direction as being due to IC emission that results from this halo (although the predicted IC intensities are in fact too high). The hard $\gamma$-ray spectrum above $\sim 300 \mathrm{MeV}$ at medium latitudes in the outer-Galaxy direction, however, cannot be explained in the framework of this classical scenario. Future work should show whether convection effects provide indeed a comfortable solution to this problem (and the similar one encountered at radio wavelengths). 


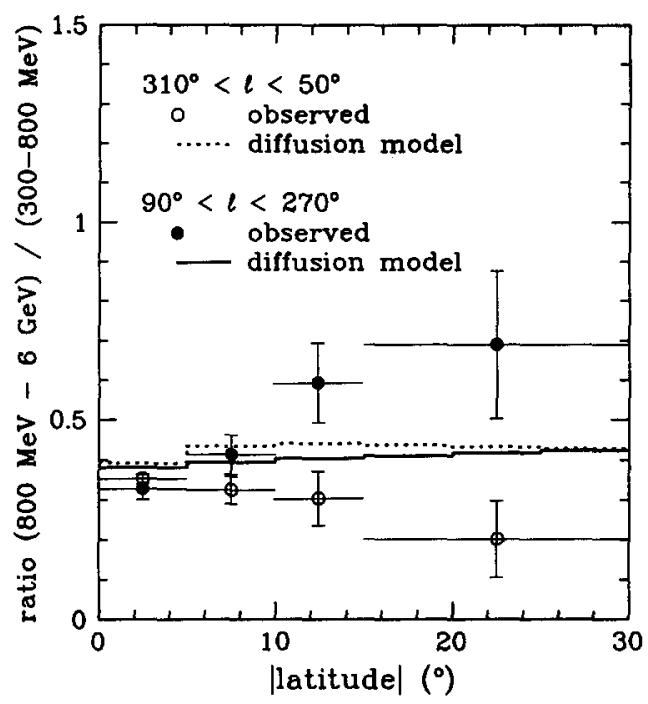

Fig. 4. Latitude distributions of the ratio between the $800 \mathrm{MeV}-6 \mathrm{GeV}$ and $300-800 \mathrm{MeV} \gamma$-ray intensities toward the inner and outer Galaxy $/ 11 /$ and the prediction from the $C R$ diffusion model $/ 6 /$.

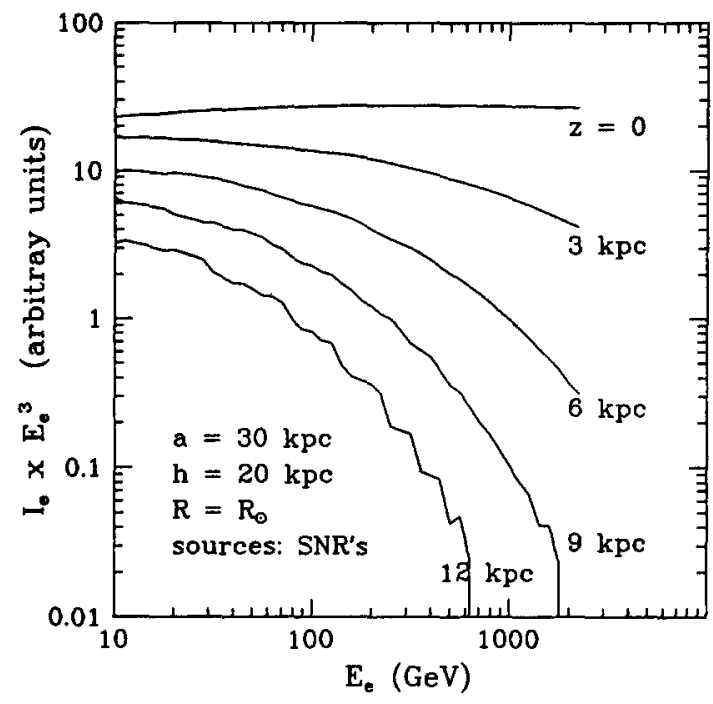

Fig. 5 High-energy electron spectra at different heights above the Galactic plane in a $C R$ diffusion model [from $/ 6 /$ ].

\section{REFERENCES}

1. V.S. Ptuskin, Hydrodynamics of the interstellar medium including cosmic rays, in Plasma Astrophysics, ESA SP-251 1986, p. 279.

2. J.B.G.M. Bloemen, On stable hydrostatic-equilibrium configurations of the Galaxy and implications for its halo Ap. J. 322, 694 (1987)

3. F. Jones, The dynamical halo and the variation of CR pathlength with energy, Ap. J. 229, 747 (1979)

4. V.L. Ginzburg and S.I. Syrovatskii, The origin of cosmic rays, Pergamon Press, Oxford, 1964.

5. F.W. Stecker and F.C. Jones, The Galactic halo question: new size constraints from Galactic $\gamma$-ray data, Ap. J. 217, 843 (1977)

6. J.B.G.M. Bloemen and V.A. Dogiel, Galactic $\gamma$-ray emission versus cosmic-ray diffusion models, Astr. Ap., submitted (1988)

7. A.W. Strong, J.B.G.M. Bloemen, T.M. Dame, I.A. Grenier, W. Hermsen, F. Lebrun, L.-A. Nyman, and P. Thaddeus, P., The radial distribution of Galactic gamma rays IV, Astr. Ap. 207, 1 (1988)

8. C.E. Fichtel and D.A. Kniffen, A study of the diffuse galactic $\gamma$ radiation, Astr. Ap. 134, 13 (1984)

9. J. Melisse and J.B.G.M. Bloemen, Astr. Ap., in prep. (1988)

10. J.B.G.M. Bloemen, Diffuse Galactic $\gamma$-ray emission, Ann. Rev. Astr. Ap. 27, in press (1989)

11. J.B.G.M. Bloemen, P. Reich, W. Reich, and R. Schlickeiser, High-energy gamma rays as a probe of cosmic-ray spectral differences throughout the Galaxy II. A comparison with radio synchrotron emission, Astr. Ap. 204, 88 (1988)

12. P. Reich and $W$. Reich, Spectral index variations of the Galactic radio continuum emission: evidence for a Galactic wind, Astr. Ap. 196, 211 (1988)

13. K.D. Lawson, C.J. Mayer, J.L. Osborne, and M.L. Parkinson, M.N.R.A.S 225, 307 (1987)

14. I. Lerche and R. Schlickeiser, On the transport and propagation of relativistic electrons in galaxies. The effect of adiabatic deceleration in a Galactic wind, Astr. Ap. 107, 148 (1982)

15. I. Lerche and $R$. Schlickeiser, On the transport and propagation of cosmic rays in galaxies. Solution of the steady-state transport equation for cosmic ray nucleons, M.N.R.A.S. 201, 1041 (1982)

16. A. J. Owens and J.R. Jokipii, Cosmic rays in a dynamical halo, Ap. J. 215, 677 (1977)

17. J. Kóta and A.J. Owens, Energy-dependent diffusion of cosmic rays in the dynamical halo model, $A p$. J. 237, 814 (1980) 